\title{
Electric vehicle power electronics cooling system pump control using fuzzy logic
}

\author{
Adnan Shaout ${ }^{*}$ and Jason Cooper \\ Electrical and Computer Engineering, University of Michigan-Dearborn \\ Received: 16-February-2017; Revised: 17-May-2017; Accepted: 20-May-2017 \\ (C)2017 ACCENTS
}

\begin{abstract}
Electric vehicles rely exclusively on electrical energy not only for propulsion, but also to power all of the vehicle subsystems. It then becomes critical that each subsystem is controlled in the most energy efficient manner possible. Some of the most energy consuming elements of a vehicle are the electric pumps that circulate coolant through an electric vehicle's coolant loop. Typically, these pumps are controlled through nothing more than simple temperature switch points, creating a sink for electrical energy. To improve the overall energy consumption, a fuzzy logic control (FLC) strategy for controlling an automotive coolant pump has been proposed. The power electronics coolant loop, which can be found in most hybrid/electric vehicles, have been examined as a temperature-based strategy which is formulated to control an industry standard pulse-width-modulated (PWM) fluid pump. Simulation of the proposed FLC has shown improvement of the overall efficiency of the coolant pump and has also reduced the overall energy consumption of the vehicle-thereby improving the electric propulsion range.
\end{abstract}

\section{Keywords}

Electric vehicle, Fuzzy logic, Power electronics cooling, PWM pump, Temperature control.

\section{Introduction}

From safety systems to advanced and alternative propulsion systems, the evolution of the automobile has been as technically advanced as any other consumer product. Over the past decade the demand for more electronically controlled mechanical systems has erupted. Although all of these Mechatronic systems are required to be functional in almost any environment, nearly all electrical and mechanical systems are affected by temperature. To make matters worse for engineers, most electrical and mechanical hardware components require a certain temperature band in order to run optimally. Thankfully, this temperature dilemma is easily solved by introducing a cooling system. Most electric vehicles use multiple coolant loops, assigning one for each component, or system of components. Fundamentally, a coolant loop consists of multiple components: (1) component to be cooled, (2) coolant pump, (3) temperature sensor(s), (4) heat exchanger, and (6) plumbing. Within such a setup it is necessary that the coolant pump circulates the coolant through the hardware. This level of control is almost strictly dependent upon the temperature of the component to be cooled.

*Author for correspondence

111
Many cooling system strategies, also take into account the state/status of the electrical component, however this additional layer of control is not relevant to the strategy outlined here as this paper will only detail a temperature-based fuzzy logic control strategy. For purposes of this paper, a standard power inverter cooling system will be evaluated as shown in Figure 1.

Given this hardware setup, a standard automotive electronic control unit (ECU) is used to control the pulse width modulated (PWM) coolant pump by commanding a certain duty cycle. The ECU employs a conventional control strategy, which directly associates a crisp input to a crisp output, meaning the number of switch points is directly related to the amount of cooling flow obtained. And so, this creates a tiered cooling strategy; more simply, the system can only command two duty cycles: (1) minimum cooling duty cycle and (2) maximum cooling duty cycle. Potentially more switch points could be introduced to create more cooling levels, but this would only create more discrete levels (with nothing in between). This strategy implies that we may get more cooling than what is actually needed. Mainly, this is due to the fact that the switch points must be defined in such a 
Adnan Shaout et al.

manner that "one-size-fits-all" for a particular cooling level.

This flaw in the control strategy has two major negative implications. First this tiered cooling level approach means more energy is being used by the pump than is really necessary. In an electric vehicle the energy consumption is critical; any energy unnecessarily used by the coolant pump is energy that is not being used to propel the vehicle, directly resulting in a decrease of electric driving range (full electric driving range is the most important feature to a potential customer).

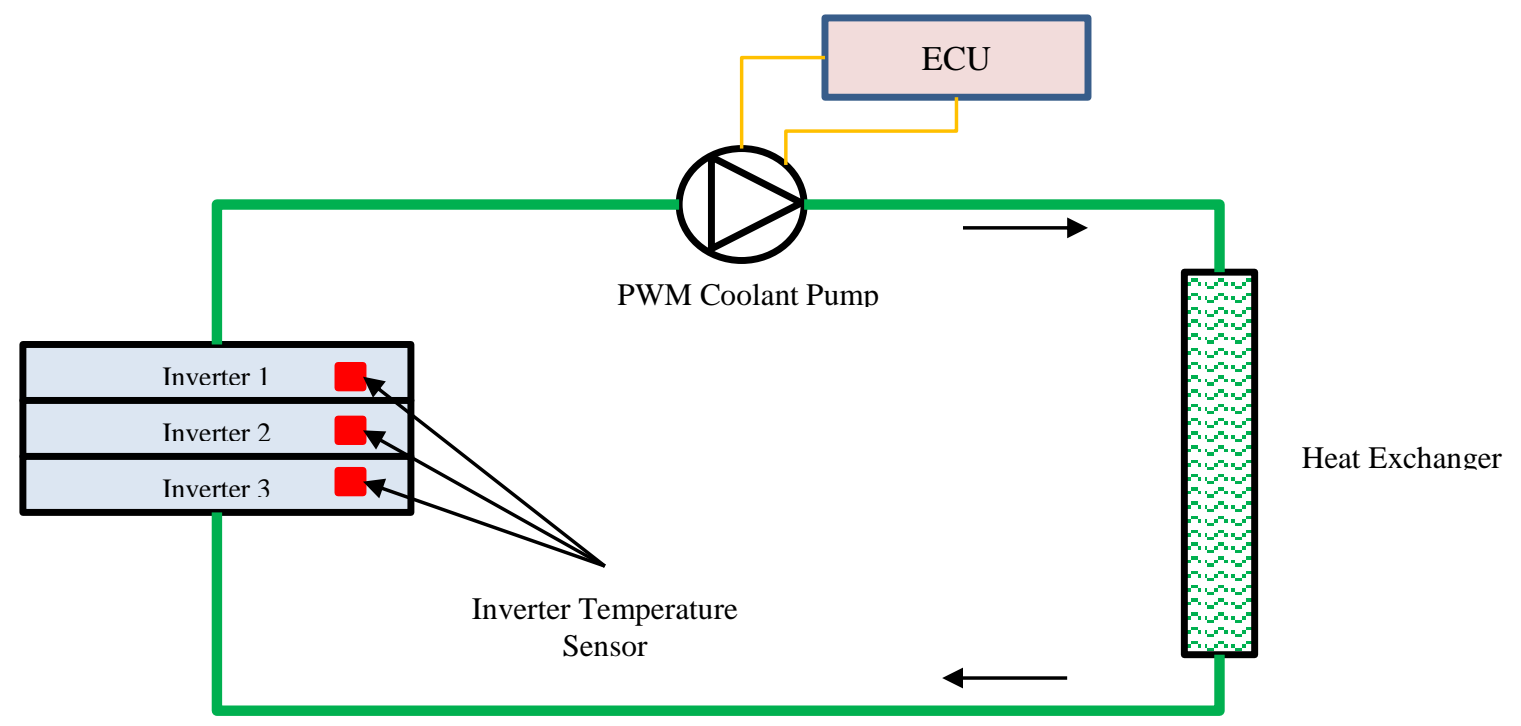

Figure 1 Power inverter coolant loop

Next, this approach causes the pump to run at much higher speeds for longer periods of time. This is mainly due to the switch points being designed low enough that higher levels of cooling are required for more scenarios (again the "one-size-fits-all" approach). Consequently the pump running faster for longer duration's causes noise, vibration, harshness (NVH) concerns. Similar to the first implication, $\mathrm{NVH}$ concerns are magnified on electric drive vehicles due to the removal of noise and vibration supplied by the internal combustion engine (ICE).

One of the best ways to resolve these two concerns is to create a smoother, more efficient pump control strategy - this is where fuzzy logic techniques come into play. Through the use of fuzzy logic, it becomes possible to use general linguistic terms such as "cold, "warm", and "hot"- as opposed to conventional control, which only has crisp on/off switch points [1]. Such a fuzzy logic control strategy will then offer more even pump control, thereby eliminating the tiered cooling structure as seen with conventional control methods. In-turn this will greatly reduce the energy consumption of our system as well as greatly reduce the $\mathrm{NVH}$ concerns.
While there are many ways of implementing a temperature-based fuzzy logic control system, it will be assumed that the hardware setup will be as outlined in Figure 1 and that a standard ECU is used to control the PWM coolant pump. The fuzzy logic control strategy detailed in this paper has been in the form of an algorithm that can later be deployed to an ECU in the form of application level software. It should also be noted that all Simulink models are for simulation purposes only, however they can be used for code/software generation, or as a building block for more complex algorithms.

\section{Literature review}

While many papers have been written about the use of fuzzy techniques to control temperature-based systems [2, 3, 4], or automotive thermal management systems [5], it is the use of this control strategy of a coolant pump for a power electronics cooling system in an electric vehicle that makes it state-of-the-art $[6,7]$. Furthermore, even though a fuzzy control strategy was devised for an automotive cooling pump, this strategy was investigated from an energy efficiency standpoint - and not necessarily a thermal management perspective. By formulating this fuzzybased control strategy for use on an electric vehicle 
we are able to reduce the overall energy consumption significantly (the exact numbers could be found through simple experimentation)-but, the truly novel part of this idea is that the energy efficiency can now be multiplied by the number of subsystems that employ this strategy (typically electric vehicles employ a minimum of two cooling loops). When all of these energy consumption numbers are put together, it should equal many miles of additional vehicle propulsion range-which in today's automotive market addresses the most critical aspect of electric vehicle production.

\section{Control methods}

From a control strategy perspective the power electronics cooling system illustrated in Figure 1 can really be broken down into two simple parts: (1) Inputs: Three inverter temperatures, as read from the three appropriately placed temperature sensors, (2) Output: Commanded Duty Cycle (see Figure 2).

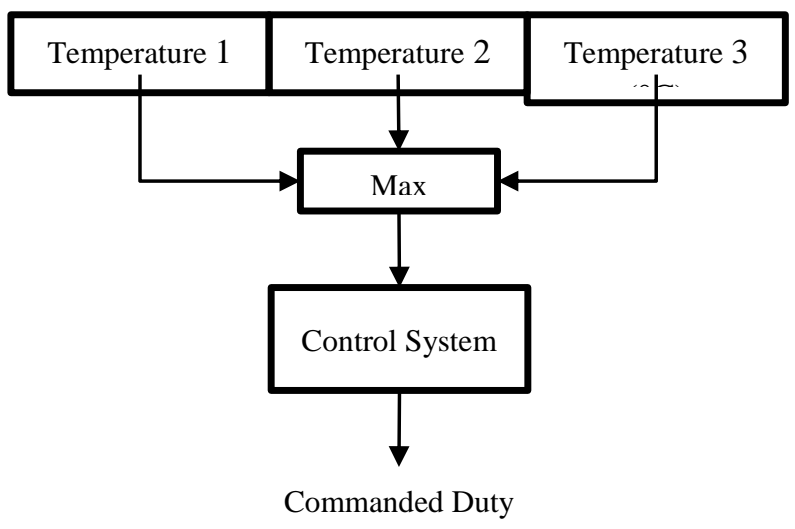

Figure 2 Inputs/outputs for inverter cooling system

From Figure 2 it is now obvious that the inputs and outputs to this system (or really any system) will be common between the conventional control strategy and the fuzzy logic control strategy. Both will have crisp temperature values as their input, and both will output a crisp duty cycle that will be used to command the pump. It is really the control systemmore specifically the techniques used within that control system-that differentiates conventional control of fuzzy logic based control [8].

\section{A. Conventional crisp control methods}

The conventional pump control method currently employed by this system is quite simple - really it is based on the concept of crisp switching points, which come directly from the temperature inputs (or rather the maximum temperature of the three temperature inputs. Even though three temperature readings are being fed into the control system, for purposes of cooling, i.e. decreasing the temperature of the inverters, it is not necessary to use all three temperatures). The maximum temperature is directly compared to two pre-defined switch points: (1) maximum cooling and (2) minimum Cooling. If the max temperature is above the minimum cooling to switch point (currently set to $35^{\circ} \mathrm{C}$ ) then the corresponding duty cycle will be commanded (currently set to 50\%). Similarly, if the $\max$ temperature is above the maximum cooling switch point $\left(50^{\circ} \mathrm{C}\right)$ then the corresponding duty cycle will be commanded (90\%).

An additional switch point is defined for each cooling level. For minimum cooling $\left(32^{\circ} \mathrm{C}\right)$, this switch point turns the pump off ( $50 \%$ to $0 \%$ ), and for maximum cooling $\left(47^{\circ} \mathrm{C}\right)$ this switch point commands the pump to the low duty cycle $(90 \%$ to $50 \%)$. Figure 3 illustrates a simple Simulink model of the described conventional crisp control method. When this simulation is run (see Figure 4), we can clearly see that the commanded pump duty cycle is directly dependent upon the temperatures being read. The afore mentioned tiered cooling levels are also clearly visible. These jumps in the commanded duty cycle are what create the inefficiencies-again causing unnecessary energy usage.

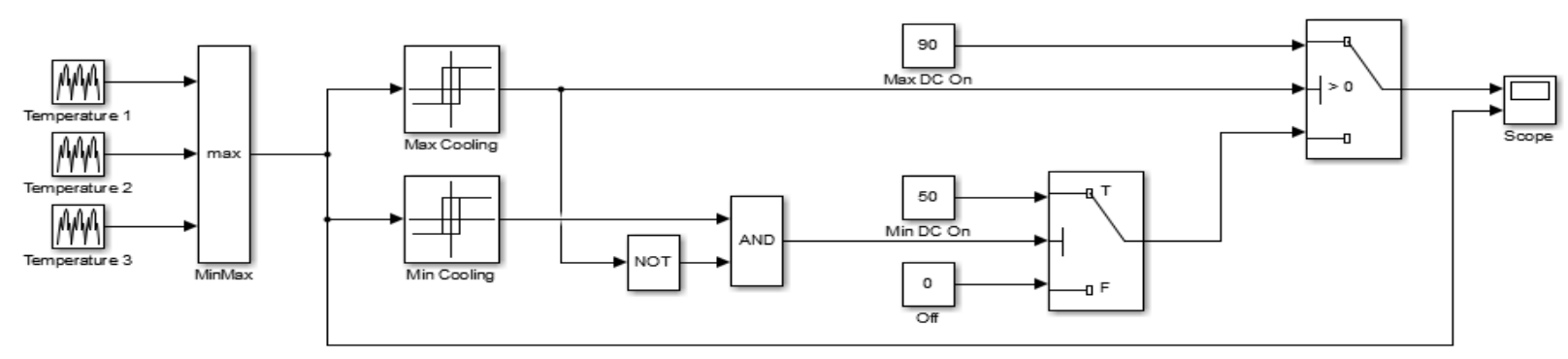

Figure 3 Simulink model for conventional crisp control 


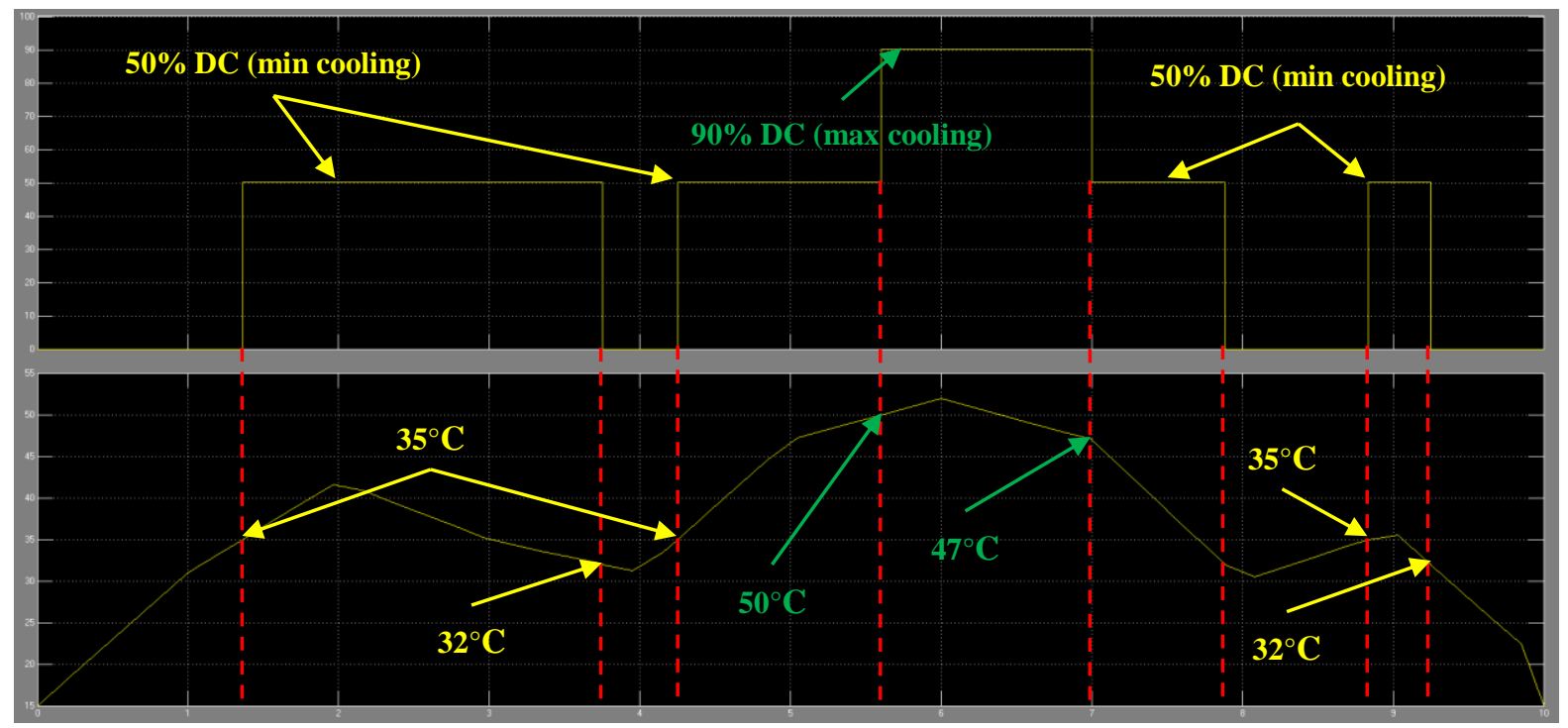

Figure 4 Pump duty cycle command (top), max temperature reading (bottom)

\section{B. Fuzzy control methods}

Fuzzy logic will allow us to generalize the conventional crisp control methods outlined in the previous section by creating non-sharp boundaries between the switch points. This will provide the flexibility needed to eliminate the duty cycle tiers visible in Figure 4. In-turn by smoothing out the coolant levels, we can lower the final duty cycle command and thereby lowering the energy consumption of the coolant pump.

Generically the fuzzy control system algorithm will consist of three main parts (Figure 5) [8,9]:

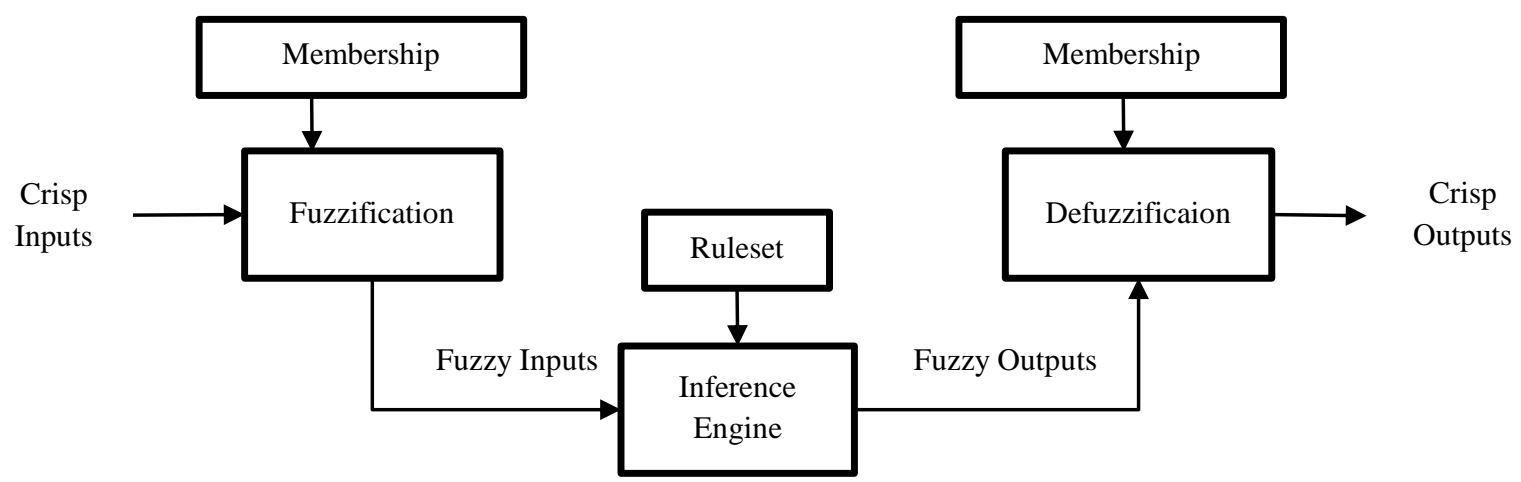

Figure 5 Block diagram of fuzzy logic algorithm

Fuzzification: The inputs are first compared to the input membership function. This allows the control algorithm to determine the degree to which the linguistic variable is true.

Inference Engine: Each rule in the fuzzy ruleset is then processed with the fuzzy input value. This process maps the input to a corresponding fuzzy linguistic output variable. Given the current input conditions, this phase of the algorithm will provide a system output.
Defuzzification: The fuzzy output is converted to a crisp value that the ECU can demand of a system.

\section{a. Crisp input/output system interfaces}

A fuzzy logic controller (FLC) really refers to the use of fuzzy logic within the control strategy itself, this then implies that the inputs, and subsequently the outputs, of the system will be identical to their conventional counterparts (we live in a crisp world!) [10]. In Figure 2 the crisp input and output variables 
were defined for use in both the conventional control methods as well as the fuzzy control methods -Table 1 again outlines the inputs and output of our system. As was the case for conventional control methods, the power inverter cooling system inputs can really be simplified to one input.

Table 1 Inputs / outputs from the inverter coolant

\begin{tabular}{cll}
\multicolumn{1}{l}{ system } & & \\
\hline \multicolumn{1}{l}{ Input } & Input type & Input unit \\
\hline$x_{1}$ & Temperature \#1 & ${ }^{\circ} \mathrm{C}$ \\
$x_{2}$ & Temperature \#2 & ${ }^{\circ} \mathrm{C}$ \\
$x_{3}$ & Temperature \#3 & ${ }^{\circ} \mathrm{C}$ \\
Output & Output Type & Output Unit \\
$y$ & Duty Cycle & $\%$
\end{tabular}

This is true since the three inputs were all temperature and all temperatures were part of the same cooling loop - more simply, only the maximum temperature of a cooling system is used since it will dictate when cooling is required. Again, as before, only the maximum temperature will be taken into account for the pump control strategy. This will allow us to define only one input variable.

The fuzzification of the input variable will start by defining it as a linguistic variable. In this case, the input variable will linguistically be denoted as temperature. The next step in the fuzzification process is creating a membership function of temperature. By partitioning it into five memberships, function envelopes, we can create overlapping regions, as seen in Figure 6. Each function will then represent a linguistic value for the linguistic variable. In this case the following linguistic values have been defined for temperature: cold, cool, warm, hot, and very hot.

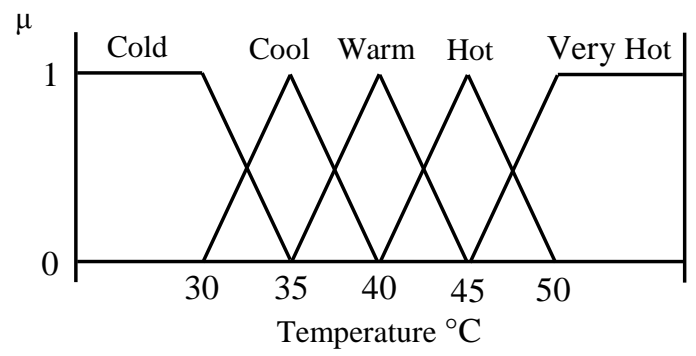

Figure 6 Membership function of linguistic input variable temperature

By overlapping the partitions, the linguistic input temperature now has a smooth transition between states - more simply put, as the temperature changes, its membership value in one function begins to decrease while it increases its membership value in 115 the next function. This technique is really a key to using fuzzy logic.

The following equations have been used to find the membership function values for each of the linguistic values:

$$
\begin{aligned}
& \mu_{\text {cold }}=\left\{\begin{array}{cc}
1 & ; \quad-\infty \leq T_{\text {in }} \leq 30 \\
\frac{35-T_{\text {in }}}{35-30} & ; \quad 30 \leq T_{\text {in }} \leq 35
\end{array}\right. \\
& \mu_{\text {cool }}= \begin{cases}\frac{T_{\text {in }}-30}{35-30} ; & 30 \leq T_{\text {in }} \leq 35 \\
\frac{40-T_{i n}}{40-35} & ; \quad 35 \leq T_{\text {in }} \leq 40\end{cases} \\
& \mu_{\text {warm }}= \begin{cases}\frac{T_{\text {in }}-35}{40-35} ; & 35 \leq T_{\text {in }} \leq 40 \\
\frac{45-T_{\text {in }}}{45-40} & ; \quad 40 \leq T_{\text {in }} \leq 45\end{cases} \\
& \mu_{\text {hot }}=\left\{\begin{array}{lll}
\frac{T_{\text {in }}-40}{45-40} & ; & 40 \leq T_{\text {in }} \leq 45 \\
\frac{50-T_{\text {in }}}{50-45} & ; & 45 \leq T_{\text {in }} \leq 50
\end{array}\right. \\
& \mu_{\text {veryhot }}=\left\{\begin{array}{ccc}
\frac{T_{\text {in }}-45}{50-45} ; & 45 \leq T_{\text {in }} \leq 50 \\
1 & ; & 50 \leq T_{\text {in }} \leq \infty
\end{array}\right.
\end{aligned}
$$

The universe of discourse was found through experimental data and is really associated with an appropriate temperature range (taking into account the data and values associated with their crisp switch point counterparts).

Universe of Discourse: $U_{\text {in }}=\{30,35,40,45,50\}$ A similar fuzzification strategy was performed on the crisp output. In this case, of course, the fuzzified output will be used to map the input to the outputafter-which it will be defuzzified to ultimately provide a crisp output duty cycle. In this system we have one output variable, which linguistically will be denoted as duty cycle.

Figure 7 shows the linguistic values which have been defined for duty cycle for very low, low, medium, high, and very high.

Universe of Discourse: $U_{\text {out }}=\{10,30,50,70,90\}$ 


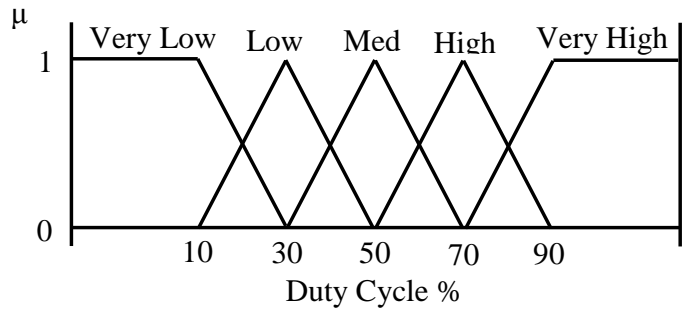

Figure 7 Membership function for linguistic input variable duty cycle

The following equations have been used to derive the output membership functions.

$$
\begin{aligned}
& \mu_{\text {verylow }}=\left\{\begin{array}{cll}
1 & ; \quad-\infty \leq D C_{\text {out }} \\
\frac{30-D C_{\text {out }}}{30-10} & ; \quad 10 \leq D C_{\text {out }} \leq 30
\end{array}\right. \\
& \mu_{\text {low }}= \begin{cases}\frac{D C_{\text {out }}-10}{30-10} & ; \quad 10 \leq D C_{\text {out }} \leq 30 \\
\frac{50-D C_{\text {out }}}{50-30} & ; \quad 30 \leq D C_{\text {out }} \leq 50\end{cases} \\
& \mu_{\text {medium }}= \begin{cases}\frac{D C_{\text {out }}-30}{50-30} & ; \quad 30 \leq D C_{\text {out }} \leq 50 \\
\frac{70-D C_{\text {out }}}{70-50} & ; \quad 50 \leq D C_{\text {out }} \leq 70\end{cases} \\
& \mu_{\text {high }}= \begin{cases}\frac{D C_{\text {out }}-50}{70-50} & ; \quad 50 \leq D C_{\text {out }} \leq 70 \\
\frac{90-D C_{\text {out }}}{90-70} & ; \quad 70 \leq D C_{\text {out }} \leq 90\end{cases} \\
& = \begin{cases}\frac{D C_{\text {out }}-45^{\mu_{\text {veryhigh }}}}{50-45} & ; 70 \leq D C_{\text {out }} \leq 90 \\
1 & ; \quad 90 \leq D C_{\text {out }} \leq \infty\end{cases}
\end{aligned}
$$

\section{b. Inference engine / rule assessment}

By defining a set of rules, the fuzzy input can be mapped to the decision that the ECU will need to make in order to properly control the pump (i.e. the fuzzy output). Typically in fuzzy logic a simple format is used when formulating the rule set: "If $X$ Then $\mathrm{Y}$ ".This format is also very convenient for the ECU that will ultimately interpret these rules to make a decision on the output [1]. Each state, or linguistic value, of the linguistic input and linguistic output variables will then have a rule associated with it.
The rule base designed for this scenario is as follows: 1. IF temperature is Cold, THEN duty cycle is Very Low.

2. IF temperature is Cool, THEN duty cycle is Low.

3. IF temperature is Warm, THEN duty cycle is Medium.

4. IF temperature is Hot, THEN duty cycle is High.

5. IF temperature is Very Hot, THEN duty cycle is Very High.

With this ruleset, we can clearly see the connection between the input variable and the output variable (both fuzzy). At each instance of time a temperature reading will intersect the input membership function and then be mapped to the output membership function (as seen in Figure 7) [10].

The analysis of Figures 6 and 7 shows that the membership functions of the linguistic input variable, temperature, and the linguistic output variable, duty cycle, are identical in shape-this was done intentionally. By defining duty cycle to have the exact same number of linguistic values as temperature we are able to ensure a one-to-one mapping between the input and output. More simply, the membership function value of the temperature is equal to the membership function of the duty cycle.

\section{c. Defuzzification}

After the rule base has been assessed we are left with a linguistic output value and a membership function value, however, since there is a one-to-one relationship between the input and output, we can simply use the membership function and the current output state to find the crisp duty cycle output. This crisp output will be used to command the cooling system pump.

The input to output membership function mapping, illustrated in Figure 8, clearly shows that the membership function value of the input is equal to the membership function value of the output. This implies that in order to defuzzify the output, we can simply use the input membership function values (as found from equations 1-5 to find the crisp duty cycle from Equations 6-10, as seen in Table 2.

The exceptions are a temperature input less than $30^{\circ} \mathrm{C}$, or greater than $50^{\circ} \mathrm{C}$. Due to hardware limitations the coolant pump cannot be commanded below $10 \%$, or beyond a $90 \%$ duty cycle, therefore any temperature below $30^{\circ} \mathrm{C}$ will default to a $0 \%$ DC and any temperature above $50^{\circ} \mathrm{C}$ will default to a 
90\% duty cycle (which can be seen in the Simulink model, as well as Figure 8 and Table 2).

It should also be noted that when multiple rules are activated, the rule with the lowest cooling request will be used to calculate the crisp duty cycle. Due to the fuzziness of the output, this technique will yield the same crisp value as using the higher cooling request (the slope equation simply changes, as seen in Equations $1-5$ and 6-10. This technique is mainly used for simplicity and modelling purposes.

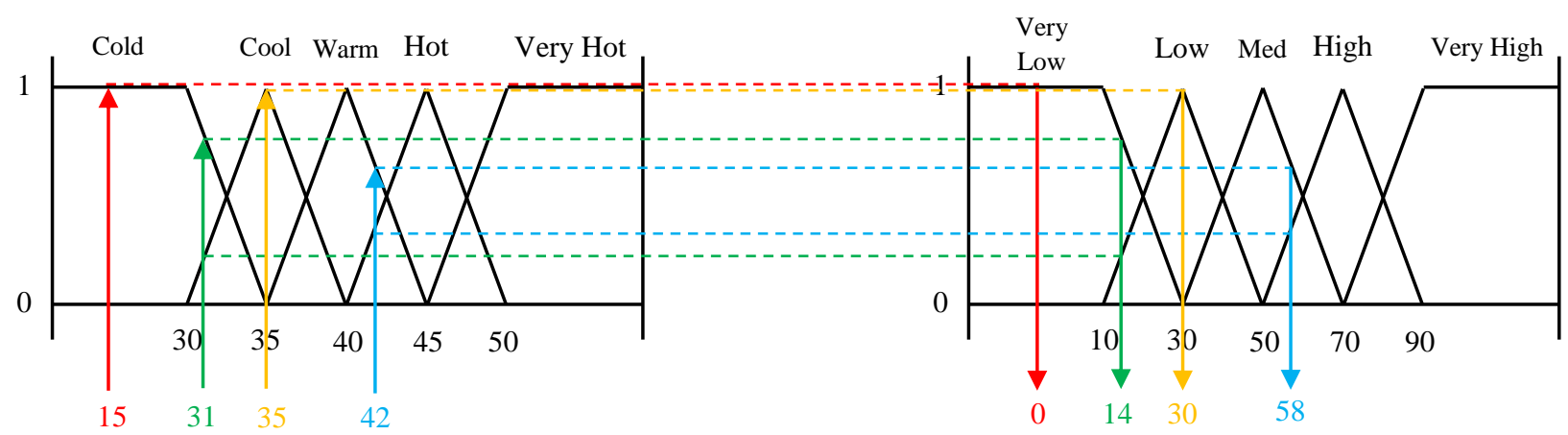

Figure 8 Mapping of a crisp temperature to a crisp duty cycle using fuzzy techniques (5 Time Samples)

Table 2 Defuzzification equations

\begin{tabular}{|c|c|c|}
\hline Temperature range $\left({ }^{\circ} \mathrm{C}\right)$ & Linguistic value & Defuzzification equation / value \\
\hline$<30$ & Cold / Very Low & 0 \\
\hline $30 \leq T_{i n} \leq 35$ & Cold / Very Low & $D C_{\text {out }}=-[\mu(30-10)-30]$ \\
\hline $35 \leq T_{\text {in }} \leq 40$ & Cool / Low & $D C_{\text {out }}=-[\mu(50-30)-50]$ \\
\hline $40 \leq T_{i n} \leq 45$ & Warm / Medium & $D C_{\text {out }}=-[\mu(70-50)-70]$ \\
\hline $45 \leq T_{i n} \leq 50$ & Hot / High & $D C_{\text {out }}=-[\mu(90-70)-90]$ \\
\hline$>50$ & Very Hot / Very High & 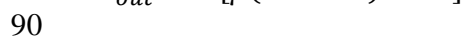 \\
\hline
\end{tabular}

\section{Simulation of fuzzy control methods}

In order to evaluate the performance of the fuzzy control strategy outlined in this paper, a Simulink model was created (see Figure 9). Simulink was chosen as the modeling tool for two main reasons: (1) it allows a user to fully model a system, which can be easily read and interpreted by outside uses, and (2) since Simulink can auto-generate controller level code, has such a model becomes useful for implementing a control strategy in an ECU using software (typically $\mathrm{C}$ code).

Similar to the simulation run using a conventional control model (Figure 3), the temperature inputs for the fuzzy numerical example/simulation will be cycled through the values seen in Table 3. Given these system inputs, a duty cycle command is found using the control strategy outlined in the preceding parts of this paper. Figure 10 and Table 3 outline the simulation results.

At every time sample the temperature value indicated is fed into the control system (as though it was read by each of the three temperature sensors). Each temperature intersects the fuzzy input membership function at least once, but typically twice. From these intersection points corresponding membership function values were found for each linguistic value. Through the rule base, each input is then mapped to a corresponding fuzzy output, which is then defuzzified using the formulas seen in the defuzzification section.

Table 3 Crisp temperature input values and crisp duty cycle output values (at each instance of time)

\begin{tabular}{|c|c|c|}
\hline $\begin{array}{l}\text { Time } \\
\text { Sample }\end{array}$ & Temperature $\left({ }^{\circ} \mathrm{C}\right)$ & Duty cycle (\%) \\
\hline 0 & 15 & 0 \\
\hline 1 & 31 & 14 \\
\hline 2 & 42 & 58 \\
\hline 3 & 35 & 30 \\
\hline 4 & 31 & 14 \\
\hline 5 & 47 & 78 \\
\hline 6 & 52 & 90 \\
\hline 7 & 47 & 78 \\
\hline 8 & 30 & 10 \\
\hline 9 & 36 & 34 \\
\hline 10 & 20 & 0 \\
\hline
\end{tabular}


Adnan Shaout et al.

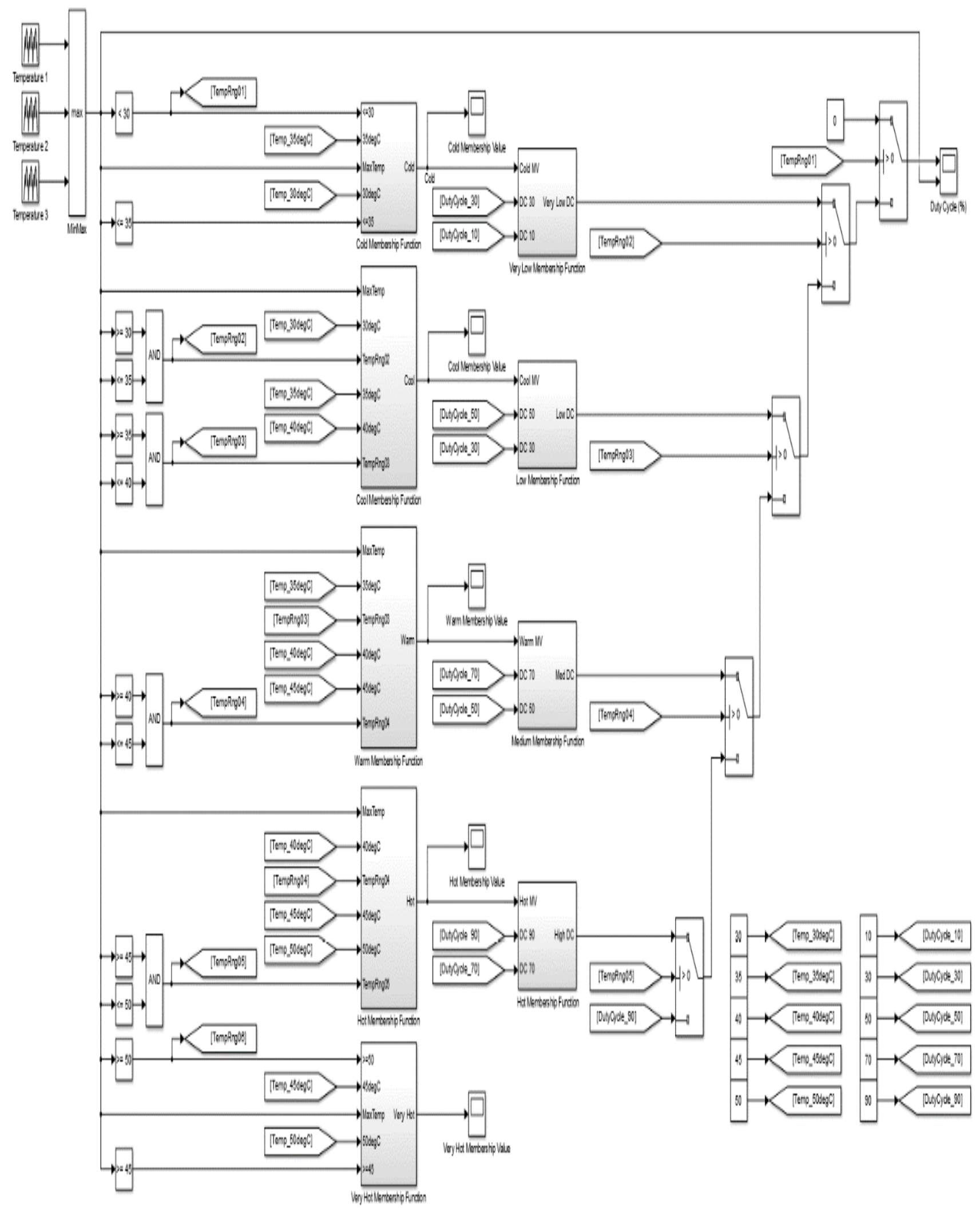

Figure 9 Simulink model for temperature-based fuzzy logic pump control 


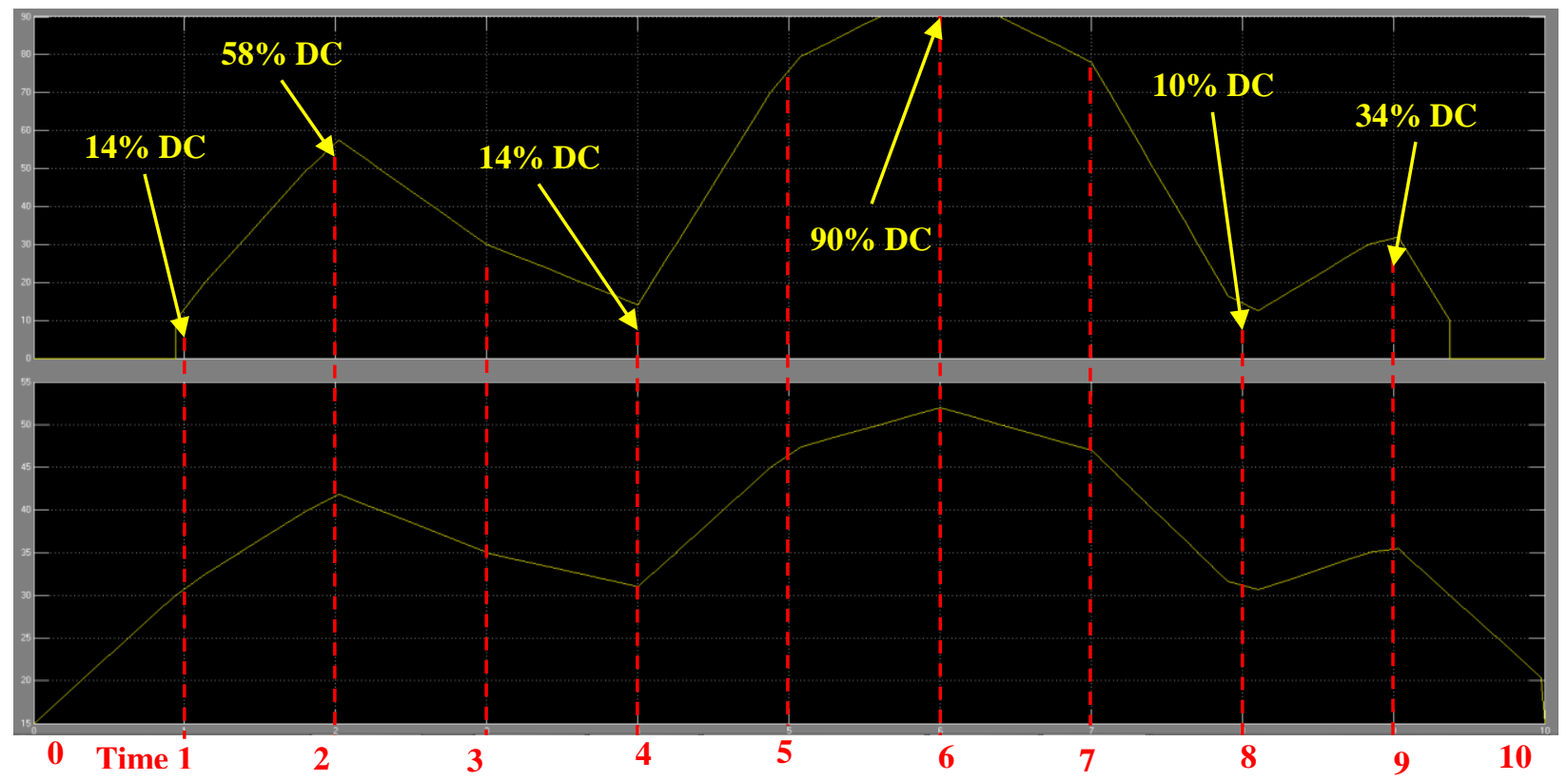

Figure 10 Duty cycle command (top), max temperature reading (bottom) for fuzzy controls

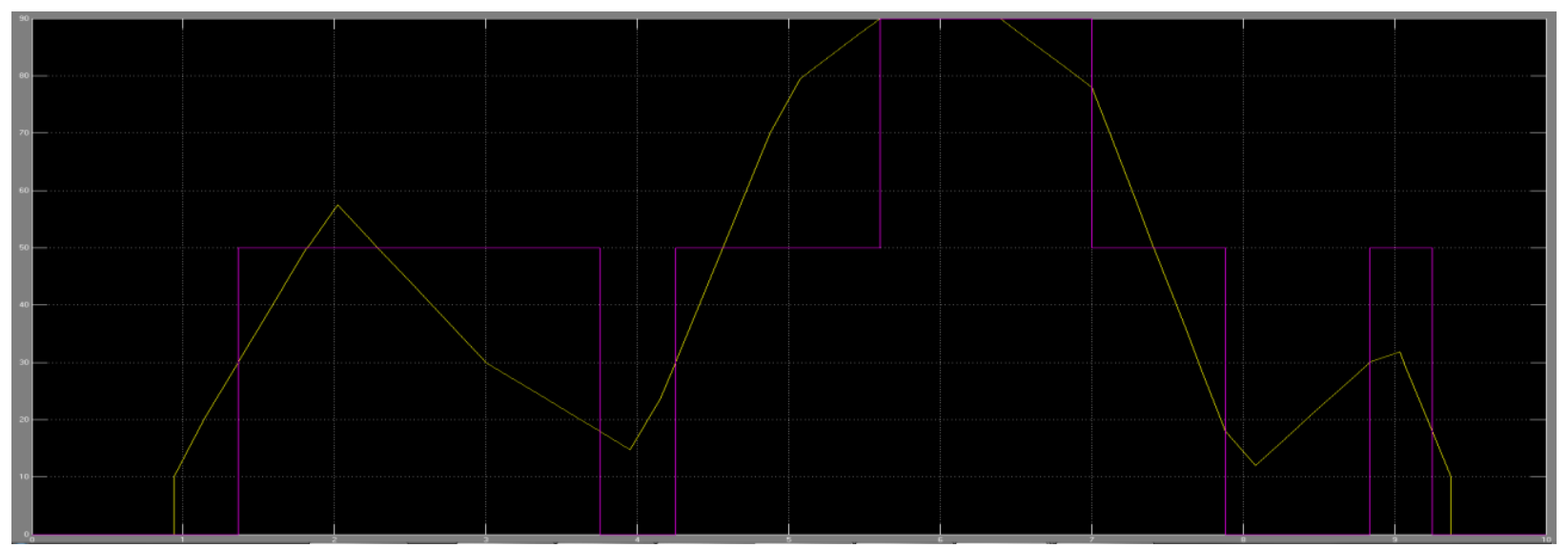

Figure 11 Comparison of simulation using conventional (purple) and fuzzy control methods (yellow)

\section{Conventional control methods vs. fuzzy control methods}

If we compare Figure 4 to Figure 10 (see Figure 11), it is very evident that the fuzzy control methods designed here were successful. The commanded duty cycle is much smoother and much more efficient when fuzzy logic techniques were employed in the control system. With the elimination of the tiered cooling levels, comes the improvement in NVH - the biggest contributor to noise and vibration is a pump that switches on and off. Furthermore, we can see that there are durations of time where the pumps are running at lower duty cycles, which of course can be directly related to the electrical energy/current consumption of the pumps. Unfortunately, there are also regions where the duty cycle is actually higher 119 with the fuzzy control strategy-this is a direct by product of using fuzzy logic. With further experimentation and tuning of the membership functions and the temperature partitions used for each linguistic value of temperature, these regions could be easily improved-and the reduction in energy consumption could be further improved.

\section{Conclusion}

Through the use of fuzzy logic techniques a control method was developed for use on the power electronics cooling system in an electric vehicle. This control strategy can not only improve the overall efficiency of the coolant pump, but it can potentially reduce the overall energy consumption of the vehicle -thereby improving the electric propulsion range. The 
NVH concerns of an electric vehicle are also addressed through the use of this strategy, which can be improved by eliminating the tiered cooling levels seen with the conventional control strategy. As seen through a Simulink simulation, the proposed fuzzybased control strategy was able to successfully smooth out the commanded duty cycle. Overall, the control strategy outlined in this paper demonstrated that it can provide a very practical solution to the two main issues associated with the power electronics coolant pump with an electric vehicle.

\section{Acknowledgment}

None.

\section{Conflicts of interest}

The authors have no conflicts of interest to declare.

\section{References}

[1] Singhala P, Shah D, Patel B. Temperature control using fuzzy logic. International Journal of Instrumentation and Control Systems. 2014; 4(1): 110.

[2] Chen Z, Mi CC. An adaptive online energy management controller for power-split HEV based on dynamic programming and fuzzy logic. In vehicle power and propulsion conference 2009 (pp. 335-9). IEEE.

[3] O'Keefe M, Bennion K. A comparison of hybrid electric vehicle power electronics cooling options. In vehicle power and propulsion conference 2007 (pp. 116-23). IEEE.

[4] Ning P, Liang Z, Wang F. Power module and cooling system thermal performance evaluation for $\mathrm{HEV}$ application. IEEE Journal of Emerging and Selected Topics in Power Electronics. 2014; 2(3):487-95.

[5] Shao C, Sun X, Wang G, Yue Y, Li X, Yang L. Numerical modeling and simulation analysis of vehicle thermal management system for a power-split hybrid electric vehicle. In international conference on electrical systems for aircraft, railway, ship propulsion and road vehicles (ESARS) 2015 (pp. 1-6). IEEE.

[6] Ibrahim BS, Aziah MA, Ahmad S, Akmeliawati R, Nizam HM, Muthalif AG, et al. Fuzzy-based temperature and humidity control for $\mathrm{HV} \mathrm{AC}$ of electric vehicle. Procedia Engineering. 2012; 41:90410.

[7] Al-Jarrah A, Salah M, Al-Tamimi A. Fuzzy logic control design for advanced vehicle thermal management system. In proceedings of the IASTED international conference on control and applications 2011 (pp. 200-4).
[8] Isizoh AN, Okide SO, Anazia AE, Ogu CD. Temperature control system using fuzzy logic technique. International Journal of Advanced Research in Artificial Intelligence. 2012; 1(3):27-31.

[9] Hanamane MD, Mudholkar RR, Jadhav BT, Sawant SR. Implementation of fuzzy temperature control using microprocessor. CSIR. 2006.

[10] Aguilar RM, Muñoz V, Callero Y. Control application using fuzzy logic: Design of a fuzzy temperature controller. INTECH Open Access Publisher; 2012.

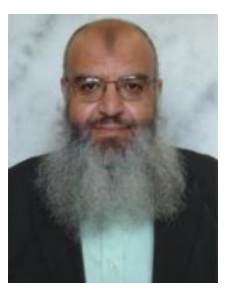

Dr. Adnan Shaout is a full professor and a Fulbright Scholar in the Computer Science Department at the Electrical and Computer Engineering Department at the University of Michigan-Dearborn. At present, he teaches courses in AI, Embedded Systems, Software Engineering, Computer Architecture, Cloud Computing, Fuzzy Logic and Engineering Applications and Computer Hardware Design. His current research is in applications of software engineering methods, cloud computing, embedded systems, fuzzy systems, real time systems and artificial intelligence. Dr. Shaout has more than 34 years of experience in teaching and conducting research in the Computer Science, Electrical and Computer Engineering fields at Syracuse University and the University of Michigan - Dearborn. Dr. Shaout has published over 210 papers in topics related to Computer Science, Electrical and Computer Engineering fields. Dr. Shaout has obtained his B.S.c, M.S. and Ph.D. in Computer Engineering from Syracuse University, Syracuse, NY, in 1982, 1983, 1987, respectively.

Email: shaout@umich.edu

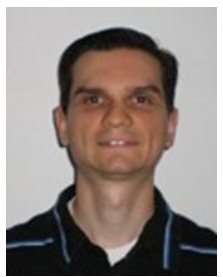

Jason Cooper is a full time algorithm and software engineer at General Motors Company. Currently, his assignments include the design and development of algorithms and software for electrification thermal management systems, as well as nextGen engine thermal management systems. Jason has over nine years of experience working the in automotive industry-this work has included the development of service diagnostics for engine controls, the design and release of ECU electronics, as well as the development of control algorithms. Jason has obtained a B.Sc. in Electrical Engineering from Michigan State University, East Lansing, MI, in 2007. He is currently a graduate student in the Department of Electrical and Computer Engineering at the University of MichiganDearborn. Jason is pursuing a M.Sc. in Electrical Engineering. 